\title{
Accurate Method for Obtaining Band Gaps in Conducting Polymers Using a DFT/Hybrid Approach
}

\author{
U. Salzner, ${ }^{*}, \uparrow$ P. G. Pickup, and R. A. Poirier \\ Department of Chemistry, Memorial University of Newfoundland, St. John's, NF AlB 3X7, Canada
}

\author{
J. B. Lagowski \\ Department of Physics and Physical Oceanography, Memorial University of Newfoundland, \\ St. John's, NF AlB 3X7, Canada
}

Received: May 16, 1997; In Final Form: August 28, 1997

\begin{abstract}
DFT calculations on a series of oligomers have been used to estimate band gaps, ionization potentials, electron affinities, and bandwidths for polyacetylene, polythiophene, polypyrrole, polythiazole, and a thiophenethiazole copolymer. Using a slightly modified hybrid functional, we obtain band gaps within $0.1 \mathrm{eV}$ of experimental solid-state values. Calculated bond lengths and bond angles for the central ring of sexithiophene differ by less than $0.026 \AA$ and $0.7^{\circ}$ from those of the sexithiophene crystal structure. IPs and EAs are overestimated by up to $0.77 \mathrm{eV}$ compared to experimental bulk values. Extrapolated bandwidths agree reasonably well with bandwidths from band structure calculations.
\end{abstract}

\section{Introduction}

Band structure calculations ${ }^{1}$ are an important theoretical tool in the search for low band gap polymers. ${ }^{2}$ Due to the size of the systems of interest, the choice of theoretical methods is, however, limited. Geometries are usually obtained at semiempirical levels such as MNDO or AM1. ${ }^{3}$ Band structures are then computed employing extended Hückel theory (EHT), ${ }^{4-6}$ the $\mathrm{Su}-\mathrm{Schrieffer}-$ Heeger model $(\mathrm{SSH}),{ }^{7}$ or the nonempirical valence electron Hamiltonian (VEH) parametrization. 8,9 Hartree-Fock (HF) band structure calculations have also been carried out, ${ }^{10}$ but the band gaps are overestimated by several electronvolts due to the problems of HF theory with the unoccupied orbital energy levels. ${ }^{11}$ Inclusion of correlation effects at the MP2 level decreases the error compared to HF theory by only about $30-50 \% .^{12,13}$ Geometry optimization at the MP2 level could decrease the error further, since HF exaggerates bond alternation.

Recent efficient implementation of density functional theory (DFT) at the local spin density approximation (LSDA) level into solid-state programs ${ }^{14-16}$ also allows inclusion of some correlation effects at the ab initio level. At the LSDA level geometries were shown to improve over those obtained with semiempirical methods and artifacts are eliminated. ${ }^{17}$ However, band gaps are severely underestimated. Since the error is fairly systematic, gaps are usually roughly doubled to obtain agreement with experiment.

In a recent publication we employed DFT/hybrid functionals to approximate the lowest allowed $\pi-\pi^{*}$-excitation energies of finite $\pi$-systems. ${ }^{18}$ DFT/hybrid functionals include gradient corrections and the exact Hartree-Fock exchange with a weight of $20 \%$. These functionals represent a considerable improve-

* Corresponding author. Tel.: (709) 737-2151. Fax: (709) 737-3702. E-mail: uli@ smaug.physics.mun.ca.

$\dagger$ Present address: Department of Chemistry, Bilkent University, 06533 Bilkent, Ankara, Turkey. Tel.: (312) 266-4000 ext. 2122. Fax: (312) 2664579. E-mail: salzner@ fen.bilent.edu.tr. ment over LSDA, as has been shown in numerous studies of molecular properties. ${ }^{19}$ We have found that HOMO-LUMO gaps are significantly closer to experiment with hybrid functionals than with LSDA, and vertical excitation energies from UV spectra were reproduced to within $0.01-0.8 \mathrm{eV} .^{18}$

In this paper, the goal was to combine the improved geometries of the DFT with the improved band gaps from hybrid functionals in a method that is more computationally expedient than solid-state band structure calculations. We use a hybrid functional to estimate band gaps $\left(E_{\mathrm{g}}\right)$, ionization potentials (IPs), electron affinities (EAs), and bandwidths. Polymer data are obtained from oligomer calculations by plotting the results against inverse chain length and extrapolating to infinity. This approach is well established for band gaps and IPs s $^{1,12,20-23}$ and is extended here to derive bandwidths. The correlation coefficients are excellent if the short-chain oligomers (up to about nine carbon atoms) are excluded. Band gaps for polyacetylene (PA), polythiophene (PTh), polypyrrole (PPy), and polythiazole (PTz) are reproduced to within $0.1 \mathrm{eV}$ of the experimental values without shifting or scaling.

DFT Orbital Energies. There is considerable controversy in the literature concerning the physical content of DFT orbital energies. Parr and Yang ${ }^{24}$ state that no simple physical meaning of Kohn-Sham orbital energies should be expected. The highest occupied orbital energy, however, equals the chemical potential and the negative of the ionization potential. ${ }^{24}$ Dreizler and Gross ${ }^{25}$ argue that the Kohn-Sham band gap would be exact for a noninteracting system. For interacting systems the correct band gap is obtained as the Kohn-Sham band gap plus the discontinuity in the exchange-correlation potential. At the LSDA level, band gaps are usually $40-50 \%$ too small. ${ }^{25}$ The question is then, whether the error is due to the approximation of the true exchange-correlation functional with a local functional or due to the neglect of the discontinuity. Theoretical analyses of LSDA and DFT energy levels led to the conclusion 
that the band gap problem is intrinsic to DFT and cannot be ameliorated by improving the functional, i.e., in going beyond LSDA. $^{26-31}$

In contrast to this, Williams and von Barth argued ${ }^{32}$ that in exact DFT the energy difference between ground and excited state as obtained by total energy differences ( $\triangle \mathrm{SCF}$ method) is equal to the difference of orbital eigenenergies plus the relaxation shift. Since this shift is negligible for extended systems, "the eigenenergies of the ground-state theory give the top of the valence band, the bottom of the conduction band, and, therefore, the energy gap". ${ }^{32}$ The underestimation of the band gap at the LSDA level was attributed to the local nature of the functional, which leads to incomplete cancellation of the self-interaction energy. Therefore, Williams and von Barth predicted that "a more accurate exchange-correlation potential could, however, considerably improve the ability of the groundstate theory to provide an approximate description of the excitation spectrum". ${ }^{32}$ These predictions are supported by a recent analysis of Kohn-Sham orbital energies by Baerends and Gritsenko, who state that "the Kohn-Sham one-electron model takes a position in DFT that is reminiscent of the one that Hartree-Fock takes in ab initio quantum chemistry. In fact, in some respects it is more fundamental since ... it is a one-electron model that is intimately related to the exact solution of the many electron problem". ${ }^{33}$ The energy of the highest occupied Kohn-Sham orbital equals the exact ionization potential, and all other virtual orbital energies are solutions in exactly the same potential as the occupied orbitals. They are therefore not upshifted in the same way as HF virtual orbitals are. $^{33}$

According to Koopmans' theorem the negative of the HOMO energy corresponds to the vertical ionization potential (IP) or the vertical excitation energy of an electron into the continuum. According to the Franck-Condon principle, the highest intensity $\left(\lambda_{\max }\right)$ in absorption spectra also corresponds to a vertical excitation since the electronic excitation is fast with respect to nuclear relaxation. For polyenes and aromatic heterocycles, the lowest allowed excitations are singlet $\pi-\pi^{*}$ transitions. Thus, the HOMO-LUMO gaps of our oligomers estimate $\lambda_{\max }$. For the polymers the situation is different. The best way to experimentally determine the $\pi-\pi^{*}$ transition energy in a solid is to use the energy of the onset of photoconductivity. This has been measured for polyacetylene and shown to be close to the absorption onset but about $0.3 \mathrm{eV}$ lower than $\lambda_{\max }$. For the polymers we therefore compare our extrapolated band gaps to the onset of absorption.

An example of a good agreement between $\lambda_{\max }$ and the experimental band gap has been observed in sexithiophene. The absorption spectrum of polycrystalline sexithiophene has been analyzed by Taliani and Blinov. ${ }^{34}$ They found that the band gap of polycrystalline sexithiophene (assigned to the first zero point in the Airy function) is $2.78 \mathrm{eV}$. This value is very close to $\lambda_{\max }=2.85 \mathrm{eV}$ of sexithiophene in solution. However Taliani and Blinov's results also showed that the lowest excitations correspond to exciton (bound electron-hole pair) and not to interband transitions. Frenkel excitons were observed at 2.36 and $2.56 \mathrm{eV}$ (a very intense band related to interband transitions was observed at $2.737 \mathrm{eV}$ ). No absorption was discovered that would correspond to the dipole-forbidden, two-photon-allowed $2 \mathrm{~A}_{\mathrm{g}}$ state. Since the extrapolation of HOMO-LUMO energy differences approximates $\pi-\pi^{*}$ band gaps, we cannot estimate the lower values of the excitation energies corresponding to (singlet and/or triplet) excitons. Excitons being neutral by nature cannot transport charge, and since we are interested in the
TABLE 1: UV Absorption Maxima in the Gas Phase and in Nonpolar Solvents for Ethylene through Octatetraene (in eV)

\begin{tabular}{lccc}
\hline & gas phase & solution & difference \\
\hline $\mathrm{C}_{2} \mathrm{H}_{4}$ & $7.08^{a}$ & $7.61^{e}$ & -0.53 \\
$\mathrm{C}_{4} \mathrm{H}_{6}$ & $5.92^{b}$ & $5.71^{e}$ & 0.21 \\
$\mathrm{C}_{6} \mathrm{H}_{8}$ & $4.95^{c}$ & $4.63^{e}$ & 0.32 \\
$\mathrm{C}_{8} \mathrm{H}_{10}$ & $4.41^{d}$ & $4.10^{e}$ & 0.31
\end{tabular}

${ }^{a} \operatorname{Ref} 55 .{ }^{b} \operatorname{Ref} 56 .{ }^{c} \operatorname{Ref} 57 .{ }^{d} \operatorname{Ref} 58 .{ }^{e} \operatorname{Ref} 59$.

minimum energy to generate free charge carriers in polymers, the property of interest is the $\pi-\pi^{*}$ band gaps and not the exciton transition energies.

\section{Computational Method}

Geometries of butadiene through $\mathrm{C}_{20} \mathrm{H}_{24}$ and of monomers through hexamers of thiophene (Th), pyrrole (Py), thiazole (Tz), and oligomers with alternating thiophene and thiazole units (ThTz) were optimized employing density functional theory. The systems were restricted to be planar, which is in agreement with the single-crystal X-ray structure of sexithiophene. ${ }^{35}$ All calculations were carried out with Gaussian 94. ${ }^{36}$

For all systems Stevens/Basch/Krauss pseudopotentials and split valence plus polarization basis sets ${ }^{37}$ (CEP-31G* keyword in Gaussian 94) $)^{36,38}$ were used. Three different functionals were employed for acetylene oligomers. The first functional corresponds to the local spin density approximation and includes the Slater exchange ${ }^{39-41}$ and the Vosko-Wilk-Nussair correlation functionals $^{42}$ (LSDA keyword in G94). ${ }^{36,38}$ The second functional is Becke's three-parameter exchange functional ${ }^{43}$ combined with Perdew's 86 correlation functional, ${ }^{44}$ which includes $20 \%$ HF exchange (B3P86 keyword in Gaussian 94). ${ }^{36,38}$ This functional provided the best estimates of energy gaps and IPs in our previous investigation of conjugated p-systems. ${ }^{18}$ However HOMO-LUMO gaps of butadiene through octatetraene are still $0.6-0.8 \mathrm{eV}$ smaller than vertical excitation energies in the gas phase. Since the precise amount of exact exchange depends on the systems studied, ${ }^{33}$ we have found that by increasing the percentage of HF exchange from $20 \%$ to $30 \%$ yielded HOMO - LUMO gaps within $\pm 0.25 \mathrm{eV}$ of the experimental data for butadiene through octatetraene. This modified functional will be referred to as B3P86-30\%. All results for PTh, PPy, PTz, and PThTz in this paper are obtained with the B3P86-30\% functional.

\section{Results and Discussion}

Band Gaps. Spectroscopic data for organic p-systems are usually determined either in solution or in the solid state (crystal or thin film). Since our calculations are for isolated molecules in the gas phase, we have attempted to first estimate the difference in excitation energies between the gas and condensed phases. Table 1 summarizes experimental absorption maxima of ethylene through octatetraene in the gas phase and in nonpolar solvents. For ethylene the difference between the gas phase and solution is largest, $0.53 \mathrm{eV}$. For butadiene through octatetraene excitation energies are about $0.2-0.3 \mathrm{eV}$ higher in the gas phase than in solution. This agrees with Brédas' findings. ${ }^{45}$

A recent comparison between one-dimensional and threedimensional band structure calculations for poly(paraphenylene vinylene) indicates that the band gap decreases by $0.2 \mathrm{eV}$ compared to results for the isolated chain if interchain interactions are accounted for. ${ }^{15}$ This shift is similar to the solvent effect described above. The UV absorption energies for sexithiophene in solution $\left(2.85^{46}\right.$ and $\left.2.86 \mathrm{eV}^{47}\right)$ and as a thin 
TABLE 2: HOMO-LUMO Gaps of $\mathrm{C}_{4} \mathrm{H}_{6}-\mathrm{C}_{20} \mathrm{H}_{24}$, Thiophene-Sexithiophene, Pyrrole-Sexipyrrole, Thiazole-Sexithiazole, and Extrapolations toward Polymer Band Gaps at B3P86-30\% in eV. Experimental $\lambda_{\max }$ Values (in Solution) Are Given in Parentheses Where Available

\begin{tabular}{|c|c|c|c|c|c|}
\hline no. of carbons & $\left(\mathrm{C}_{n} \mathrm{H}_{n+2}\right)_{x}$ & (thiophene) $)_{x}$ & $(\text { pyrrole })_{x}$ & $(\text { thiazole })_{x}$ & $(\text { thiophene/thiazole) })_{x}$ \\
\hline 4 & $6.15(5.71)^{a}$ & $6.73(5.23)^{d}$ & $7.44(5.97)^{h}$ & $6.76(5.14)^{j, k}$ & \\
\hline 6 & $5.03(4.63)^{a}$ & & & & \\
\hline 8 & $4.33(4.10)^{a}$ & $4.80(4.05)^{e}$ & $5.49(4.49)^{h}$ & 4.92 & \\
\hline 10 & $3.84(3.71)^{a}$ & & & & \\
\hline 12 & $3.49(3.41)^{a}$ & $4.00(3.49)^{e}$ & $4.73(3.91)^{h}$ & 4.20 & 4.10 \\
\hline 14 & $3.22(3.18)^{a}$ & & & & \\
\hline 16 & $3.01(3.02)^{a}$ & $3.58(3.16)^{e}$ & 4.34 & 3.80 & 3.69 \\
\hline 18 & $2.85(2.82)^{a}$ & & & & \\
\hline 20 & $2.71(2.77)^{a}$ & $3.32(2.99)^{e}$ & $4.10(3.38)^{h}$ & 3.55 & 3.45 \\
\hline 22 & & & & & \\
\hline 24 & $2.51(2.61)^{a}$ & $3.15(2.85)^{e}$ & 3.95 & 3.40 & 3.29 \\
\hline 28 & & & $(3.25)^{h}$ & & \\
\hline 8 & $1.57(1.83)$ & $2.30(2.27)$ & $3.16(2.71)$ & 2.59 & 2.47 \\
\hline exptl solid & $1.48^{b}$ & $2.0^{f}$ & $2.85^{h}$ & $2.47^{j, k}$ & \\
\hline VEH & $1.43^{c}$ & $1.72^{c}$ & $3.6^{i}$ & & \\
\hline LSDA & & $1.3^{g}$ & $1.9^{g}$ & & \\
\hline
\end{tabular}

${ }^{a} \operatorname{Ref} 59 .{ }^{b} \operatorname{Ref} 48 .{ }^{c} \operatorname{Ref} 1 .{ }^{d} \operatorname{Ref} 60 .{ }^{e} \operatorname{Ref} 46 .{ }^{f} \operatorname{Ref} 49 .{ }^{g} \operatorname{Ref} 14 .{ }^{h} \operatorname{Ref} 23 .{ }^{i} \operatorname{Ref} 22 .{ }^{j} \operatorname{Ref} 50 .{ }^{k}$ Experimental results are obtained for 3 -methyl derivatives.

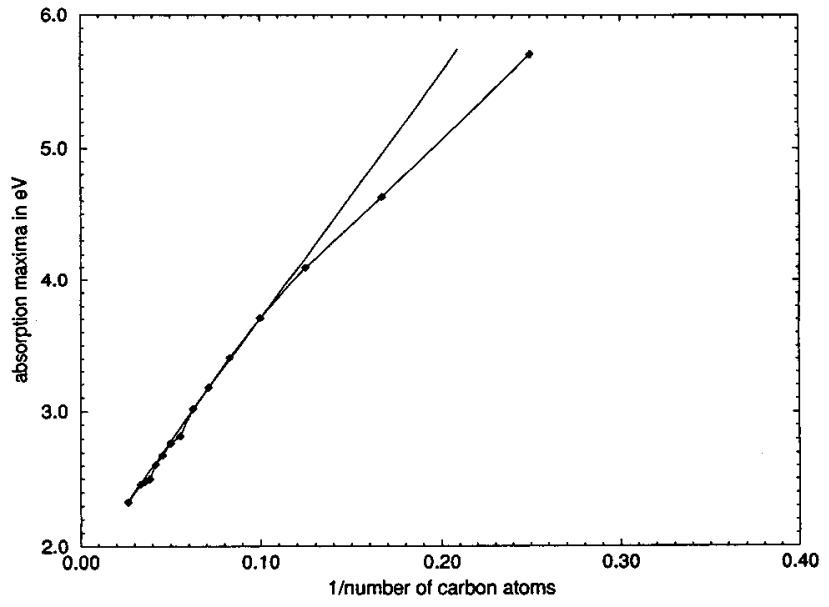

Figure 1. Experimental $\lambda_{\max }$ vs inverse chain length for $\mathrm{C}_{4} \mathrm{H}_{6}$ through $\mathrm{C}_{38} \mathrm{H}_{40}$.

film $(2.78 \mathrm{eV})^{34}$ differ by only $0.07 \mathrm{eV}$. We infer from these findings that excitation energies in solution are similar to those in the solid state. Gas-phase data appear to be roughly $0.2-$ $0.3 \mathrm{eV}$ higher than condensed-phase results.

Table 2 lists HOMO-LUMO gaps for acetylene, thiophene, pyrrole, thiazole, and mixed thiophene-thiazole oligomers. Experimental data are included where available. Figure 1 shows a plot of experimental absorption maxima in solution against inverse chain length for butadiene through $\mathrm{C}_{20} \mathrm{H}_{24}$. There exists a good linear correlation if small oligomers (with less than 10 carbon atoms), which deviate considerably from a straight line, are excluded. Our theoretical data (not shown) exhibit a similar trend.

In Figure 2 theoretical HOMO-LUMO gaps (at LSDA, $\mathrm{B} 3 \mathrm{P} 86$, and $\mathrm{B} 3 \mathrm{P} 86-30 \%)$ and experimental UV absorption maxima for $\mathrm{C}_{10} \mathrm{H}_{12}$ through $\mathrm{C}_{20} \mathrm{H}_{24}$ are plotted against inverse chain length. It can be seen that with the DFT/hybrid functionals theoretical values decrease more rapidly than the experimental data. The slopes are exptl, $19.31 \mathrm{eV} \times N_{\mathrm{C}}$ (number of carbons); B3P86, $21.22 \mathrm{eV} \times N_{\mathrm{C}}$; B3P86-30\%, $22.62 \mathrm{eV} \times N_{\mathrm{C}}$. At the LSDA level the slope of $16.21 \mathrm{eV} \times$ $N_{\mathrm{C}}$ is smaller than the experimental slope. This means that there is a difference in accuracy of energy gaps for small and large oligomers. Several factors might contribute to this. The difference between excitation energies obtained with the $\triangle \mathrm{SCF}$

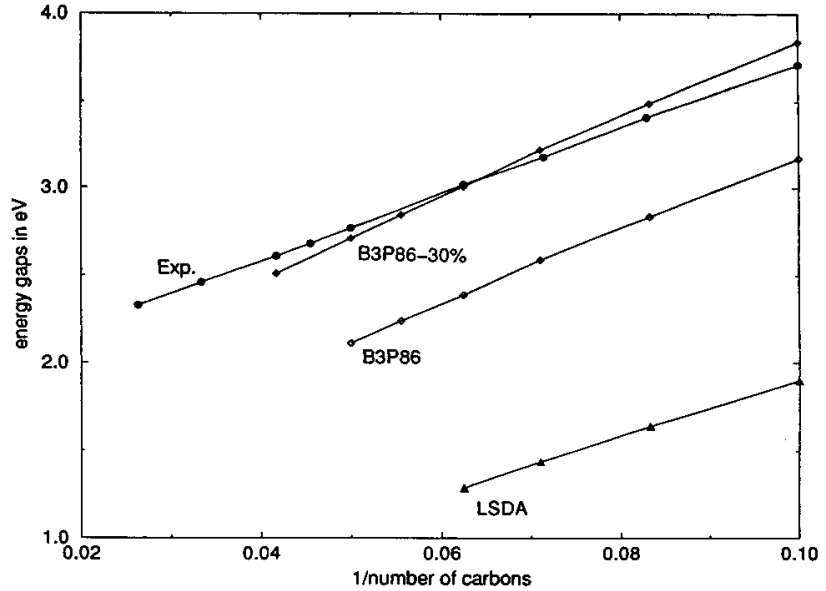

Figure 2. Experimental $\lambda_{\max }$ and theoretical HOMO-LUMO gaps at LSDA, B3P86, and B3P86-30\% vs inverse chain length for $\mathrm{C}_{10} \mathrm{H}_{12}$ through $\mathrm{C}_{38} \mathrm{H}_{40}$.

method and those approximated as orbital energy differences is the relaxation shift. ${ }^{32}$ This relaxation shift is negligible for extended systems. However, the oligomers studied here are rather small. So we might see the decrease in the relaxation shift as the oligomers become larger. Apart from this, the experimental solution data might be affected by disorder. The slope might therefore be smaller since the chains are not necessarily perfectly linear and planar. Nonetheless, the errors due to the different chain length dependencies are quite small compared to the underestimation of the gaps at the LSDA and B3P86 levels. Including $30 \%$ of $\mathrm{HF}$ exchange leads to a substantial improvement in the energy gaps.

Figures 3 and 4 show vertical excitation energies in solution and HOMO-LUMO gaps at the B3P86-30\% level for thiophene through sexithiophene and for pyrrole through heptapyrrole. Experimental data are taken from Colditz et al. ${ }^{46}$ and Zotti et al., ${ }^{23}$ respectively. Again theoretical and experimental data show that monomers do not lie on the straight lines going through the other points for the larger oligomers. (Zotti et al. included the pyrrole monomer in the extrapolation and estimated the band gap of a hypothetical perfectly ordered polypyrrole to be 0.1 eV larger than our estimate.) Bithiophene deviates only slightly while the correlation is good for bipyrrole. As with the polyenes, agreement between theory and experiment improves as the chain lengths increase. Considering the expected $0.2 \mathrm{eV}$ 


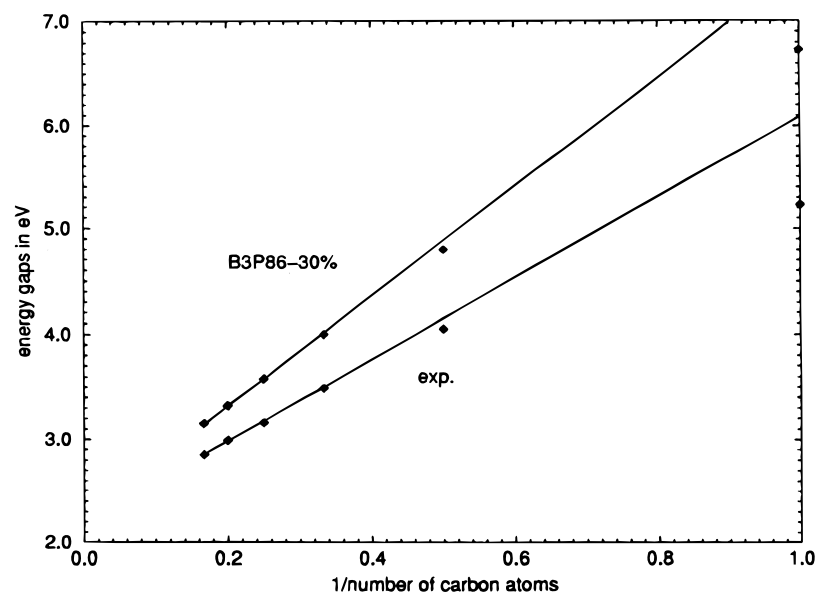

Figure 3. Experimental $\lambda_{\max }$ and B3P86-30\% HOMO-LUMO gaps vs inverse chain length for thiophene through sexithiophene.

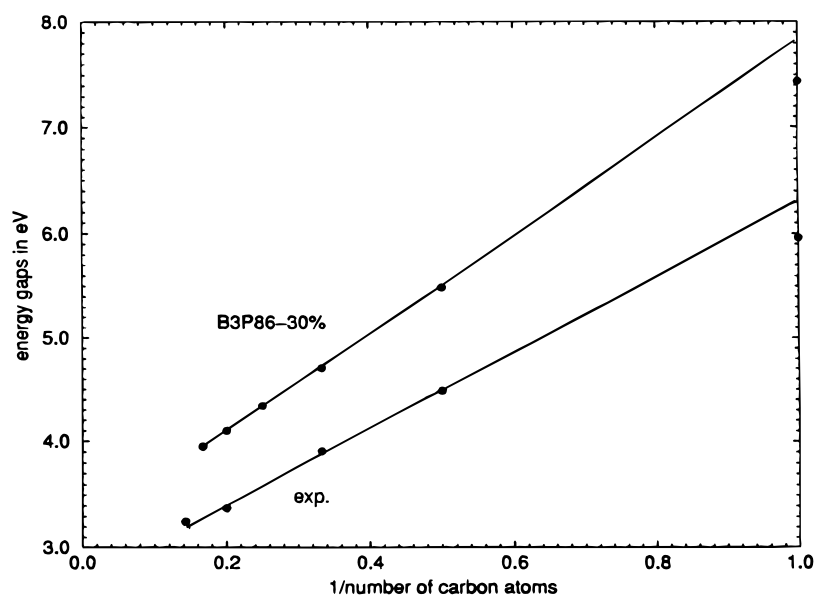

Figure 4. Experimental $\lambda_{\max }$ and B3P86-30\% HOMO-LUMO gaps vs inverse chain length for pyrrole through heptapyrrole.

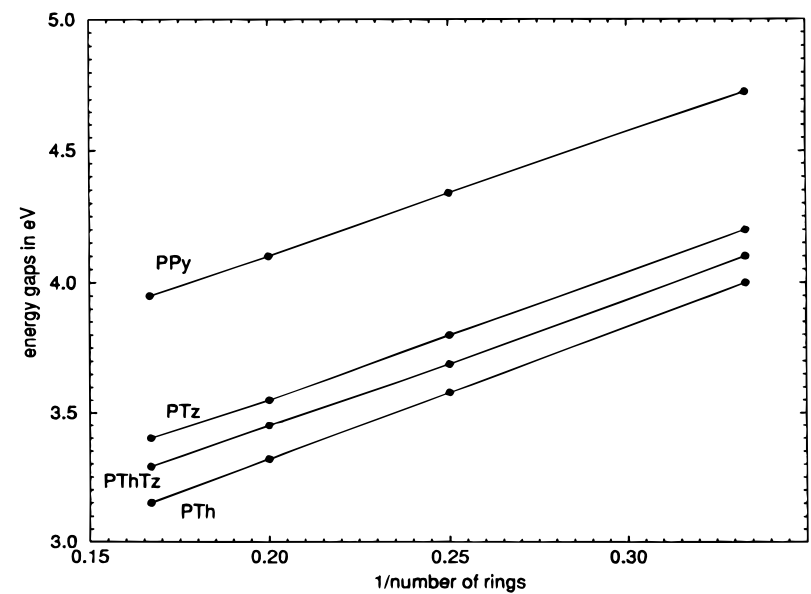

Figure 5. HOMO-LUMO gaps vs inverse number of rings for thiophene, pyrrole, thiazole, and thiophene-thiazole oligomers at B3P86-30\%.

difference between gas-phase and condensed-phase results, agreement between theory and experiment is excellent for the larger oligomers.

The numbers for Th, Py, Tz, and ThTz oligomers with 10 carbons or more are plotted against inverse chain length in Figure 5 and extrapolated to infinity. The correlation coefficients are 1.000 in every case. The extrapolated band gap values are between 0.1 and $0.3 \mathrm{eV}$ higher than experimental gaps for the polymers (PA, $1.57 \mathrm{vs} 1.48 \mathrm{eV} ;{ }^{48} \mathrm{PTh}, 2.30 \mathrm{vs} 2.0$

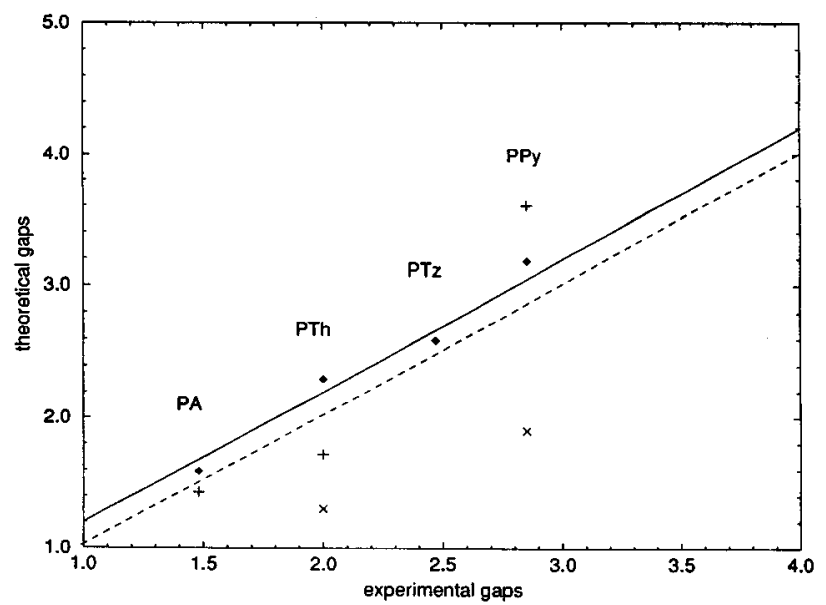

Figure 6. Extrapolated band gaps at B3P86-30\% (filled diamonds), band gaps from band structure calculations at VEH (plus signs), and LSDA ( $\times$ signs) vs experimental band gaps for PA, PTh, PPy, and Tz. The dashed line indicates one-to-one correspondence between theory and experiment. The solid line is shifted up by $0.2 \mathrm{eV}$, which is the expected difference between gas-phase and condensed-phase data.

TABLE 3: Comparison between Experimental and Theoretical IPs for Ethylene through Octatetraene, Thiophene, Bithiophene, and Pyrrole

\begin{tabular}{lccc}
\hline & exptl & B3P86-30\% & error \\
\hline $\mathrm{C}_{2} \mathrm{H}_{4}$ & $10.63^{a}$ & 8.32 & 2.32 \\
$\mathrm{C}_{4} \mathrm{H}_{6}$ & $9.09^{a}$ & 7.29 & 1.80 \\
$\mathrm{C}_{6} \mathrm{H}_{8}$ & $8.2^{a}$ & 6.74 & 1.46 \\
$\mathrm{C}_{8} \mathrm{H}_{10}$ & $7.80^{b}$ & 6.38 & 1.42 \\
infinite chain & & & 1.0 \\
thiophene & $8.85^{c}$ & 7.48 & 1.37 \\
pyrrole & $8.21^{d}$ & 6.65 & 1.56 \\
bithiophene & $7.63^{e}$ & 6.59 & 1.04
\end{tabular}

${ }^{a} \operatorname{Ref} 61 .{ }^{b} \operatorname{Ref} 58 .{ }^{c} \operatorname{Ref} 62 .{ }^{d} \operatorname{Ref} 63 .{ }^{e} \operatorname{Ref} 64$.

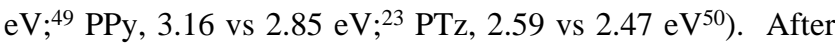
taking into account that condensed phase values are about 0.2 $\mathrm{eV}$ smaller than gas-phase data, experimental band gaps are reproduced within $\pm 0.1 \mathrm{eV}$. This accuracy is much better than with band structure calculations employing VEH and LSDA (Figure 6).

Ionization Potentials and Electron Affinities. In Table 3 negative HOMO energies at the B3P86-30\% level are compared to experimental IPs for ethylene through octatetraene, thiophene, pyrrole, and bithiophene. Theoretical IPs are generally too low. Again agreement between theory and experiment improves with increasing molecular size. In Figure 7 the errors for the polyene IPs are plotted against inverse chain length. Extrapolation (ethylene excluded) leads to an upper bound of about $1 \mathrm{eV}$ for the error expected for the IP of PA. The reason for the underestimation of the IPs is probably related to the incomplete self-interaction cancellation in LSDA. This was shown to elevate energy levels. ${ }^{32}$ Although hybrid functionals represent a considerable improvement over LSDA, the cancellation might still be incomplete.

Figures 8 and 9 show plots of IPs and EAs against inverse chain lengths for acetylene, thiophene, pyrrole, thiazole, and thiophene-thiazole oligomers. The correlations are very good. Only for the copolymer between thiophene and thiazole is the correlation not as good, but this is due to the fact that the threeand five-membered oligomers are obtained by adding just one thiophene ring instead of the proper thiophene-thiazole repeat unit. Since the deviations are small, this does not compromise the results. Extrapolated IPs and EAs are summarized and compared with experimental data in Table 4. 


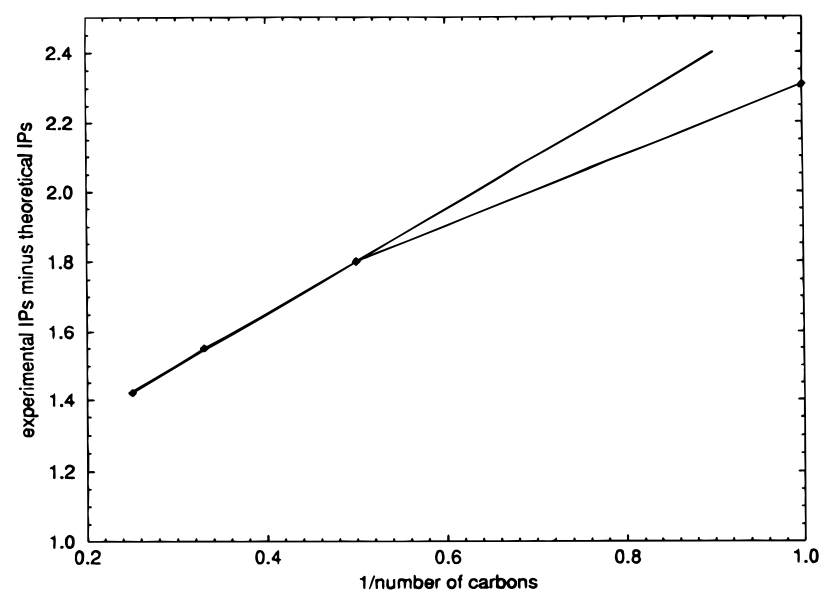

Figure 7. Differences between experimental and theoretical IPs vs inverse chain lengths for ethylene through octatetraene.

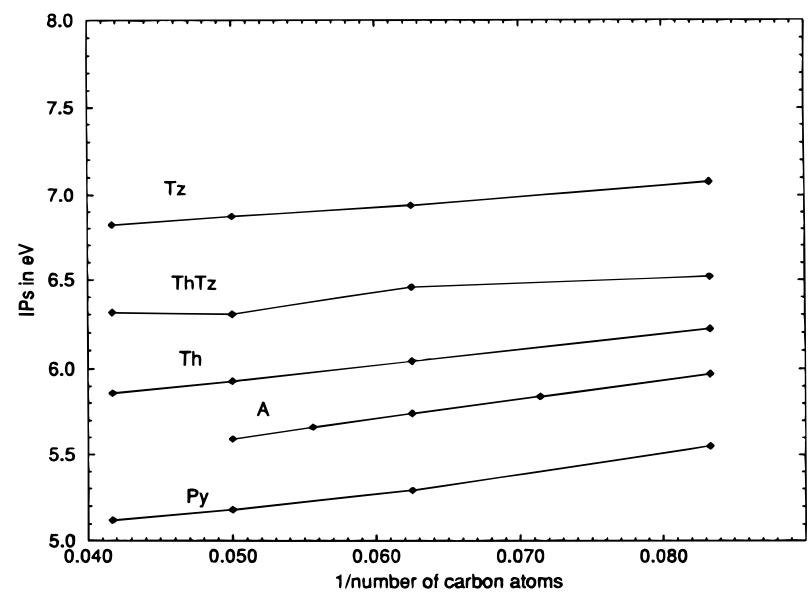

Figure 8. Theoretical negative HOMO energies (IPs) at B3P86-30\% vs inverse chain lengths for acetylene, thiophene, pyrrole, thiazole, and thiophene-thiazole oligomers.

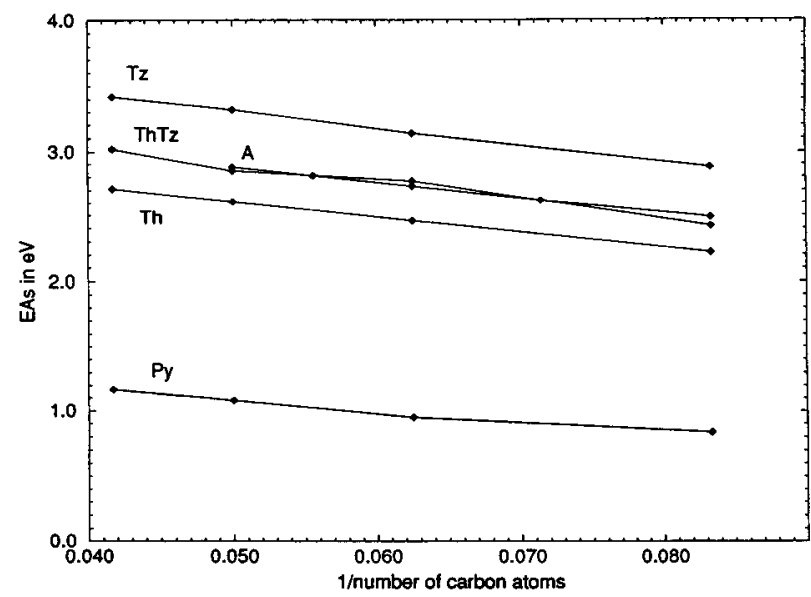

Figure 9. Theoretical negative LUMO energies (EAs) at B3P86-30\% vs inverse chain lengths for acetylene, thiophene, pyrrole, thiazole, and thiophene-thiazole oligomers.

Although our oligomer data are underestimated, extrapolated theoretical IPs for the polymers are between 0.50 and $0.77 \mathrm{eV}$ higher than those measured for polymers in the solid state. This can be explained by the fact that there is an experimentally determined $\sim 1.7 \mathrm{eV}$ difference between IPs of isolated molecules in the gas phase and IPs of molecular crystals. ${ }^{51}$ This polarization energy of organic solids was shown to be independent of the size of the system and should therefore be similar
TABLE 4: Comparison between Experimental and Theoretical IPs and EAs for PA, PTh, PPy, PTz, and PThTz in eV

\begin{tabular}{llllll}
\hline & \multicolumn{1}{c}{ PA } & PTh & PPy & PTz & PThTz \\
\hline theor & 5.02 & 5.50 & 4.77 & 6.56 & 6.06 \\
exptl & $4.5 \pm 1^{a}$ & $5.0^{b}$ & $4.0^{b}$ & & \\
& \multicolumn{5}{c}{ EAs } \\
theor & 3.45 & 3.20 & 1.61 & 3.97 & 3.58 \\
exptc $^{c}$ & 3.0 & 3.0 & 0.8 &
\end{tabular}

${ }^{a}$ Ref $65 .{ }^{b}$ Ref 22. ${ }^{c}$ Experimental EAs are obtained as the difference between experimental IPs and gaps.

TABLE 5: Extrapolated Bandwidths at B3P86-30\% and Bandwidth from Band Structure Calculations at the VEH, LSDA, HF, and MP2 Levels of Theory Where Available for PA, PTh, PPy, PTz, and PThTz

\begin{tabular}{|c|c|c|c|c|c|}
\hline & PA & PTh & PPy & $\mathrm{PTz}$ & PThTz \\
\hline \multicolumn{6}{|c|}{ Valence Band } \\
\hline В3Р86-30\% & 6.02 & 2.75 & 3.08 & 2.92 & 2.51 \\
\hline VEH & $6.5^{a}$ & $2.6^{a}$ & $3.8^{a}$ & & \\
\hline LSDA & & $2.4^{b, e}$ & & & \\
\hline $\mathrm{HF}$ & $7.826^{c}$ & $2.890^{c}$ & $3.905^{d}$ & & \\
\hline $\mathrm{MP}^{d}$ & & 3.07 & 3.95 & & \\
\hline \multicolumn{6}{|c|}{ Conduction Band } \\
\hline В3Р $86-30 \%$ & 5.22 & 4.42 & 2.98 & 4.04 & 3.26 \\
\hline LSDA & & $3.0^{b}$ & & & \\
\hline $\mathrm{HF}$ & $9.330^{c}$ & $3.246^{\circ}$ & $1.777^{d}$ & & \\
\hline $\mathrm{MP}^{d}$ & & 3.494 & 1.899 & & \\
\hline
\end{tabular}

${ }^{a} \operatorname{Ref} 22 .{ }^{b} \operatorname{Ref} 17 .{ }^{c} \operatorname{Ref} 10 .{ }^{d} \operatorname{Ref} 13 .{ }^{e}$ In Ref 17 the valence bandwidth is quoted to be $3.7 \mathrm{eV}$. Comparison with Figure $2^{17}$ seems to indicate that the HOMO plus HOMO-1 bands are included in this value. The width of the actual valence band appears to be about 2.4 $\mathrm{eV}$.

for organic polymers. ${ }^{51}$ Hence, extrapolation of data (theoretical and experimental) obtained for oligomers in the gas phase yields IPs for polymers that are intrinsically $1.7 \mathrm{eV}$ too high. We estimated that with our approach extrapolated IPs for polymers are systematically underestimated by about $1 \mathrm{eV}$. Therefore, we overestimate bulk polymer data by only $0.77 \mathrm{eV}$ (instead of $1.7 \mathrm{eV}$ ). Since both the polarization energy and the error in our approach are roughly constant, we can obtain reasonable estimates of the IPs of solids. Especially trends in IPs are expected to be reproduced reliably. PTz, for instance, is theoretically predicted to have a $1.06 \mathrm{eV}$ higher IP and a 0.77 $\mathrm{eV}$ higher EA than PTh. This is consistent with the experimental finding that PTz can be $n$-doped but not $\mathrm{p}$-doped, ${ }^{50}$ while PTh can easily be p-doped.

Experimental EAs of PA, PTh, PPy, and PTz are not available. Estimates can be obtained by subtracting experimental gaps form experimental IPs. These numbers are listed as experimental EAs in Table 4. EAs are obtained with similar errors as IPs $(0.5-0.8 \mathrm{eV})$. This has to be the case since gaps that are predicted accurately are the difference between IPs and EAs.

Bandwidths. In addition to band gaps and band edges, band structure calculations also provide bandwidths. Within the oneelectron approximation bandwidths are too crude to simulate optical spectra, but they provide a rough guide for the degree of delocalization in the system. In Table 5 theoretical bandwidths obtained from extrapolation of the appropriate oligomer energy levels are compared to bandwidths from band structure calculations. The agreement between our and VEH results ${ }^{22}$ is good for the conduction and the valence bands of polyacetylene. $\mathrm{VEH}$ is known to slightly overestimate bandwidths since the top of the valence band is reproduced very well but the bottom 
TABLE 6: Geometries of the Inner Rings of Sexithiophene and Sexipyrrole at B3P86-30\% Compared to Experiment and LSDA Optimized Structures of Polymers

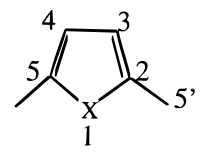

\begin{tabular}{|c|c|c|c|c|c|c|c|}
\hline & \multicolumn{4}{|c|}{ thiophene } & \multicolumn{3}{|c|}{ pyrrole } \\
\hline & $\begin{array}{c}\text { В3Р86-30\% } \\
6-\mathrm{T}\end{array}$ & $\begin{array}{c}\operatorname{exptl}^{a} \\
6-\mathrm{T}\end{array}$ & $\begin{array}{c}\mathrm{LSDA}^{b} \\
\text { PTh }\end{array}$ & $\mathrm{HF}^{c}$ & $\begin{array}{c}\text { В3Р86-30\% } \\
6-\mathrm{P}\end{array}$ & $\begin{array}{c}\mathrm{LSDA}^{b} \\
\mathrm{PPy}\end{array}$ & $\mathrm{HF}^{c}$ \\
\hline $1-2$ & 1.752 & 1.73 & 1.729 & 1.745 & 1.380 & 1.406 & 1.347 \\
\hline $2-3$ & 1.396 & 1.37 & 1.395 & 1.358 & 1.402 & 1.405 & 1.371 \\
\hline $3-4$ & 1.427 & 1.41 & 1.428 & 1.427 & 1.429 & 1.433 & 1.425 \\
\hline $2-5$ & 1.459 & 1.45 & 1.461 & 1.454 & 1.457 & 1.462 & 1.449 \\
\hline $2-1-5$ & 92.5 & 91.9 & 93.4 & 92.1 & 111.1 & 111.0 & 110.7 \\
\hline $1-2-3$ & 110.4 & 111 & 110.4 & 110.4 & 106.9 & 106.2 & 106.9 \\
\hline $2-3-4$ & 113.3 & 114 & 112.9 & 113.5 & 107.7 & 108.3 & 107.7 \\
\hline $3-2-5^{\prime}$ & 128.9 & 129 & 129.5 & 128.6 & 131.3 & 132.2 & 113.0 \\
\hline
\end{tabular}

${ }^{a} \operatorname{Ref} 35 .{ }^{b} \operatorname{Ref} 14 .^{c}$ Refs $12,13$.

of the valence band is too low. ${ }^{8}$ It is therefore encouraging that our method yields slightly smaller bandwidths than VEH for PA. For the PTh valence band, our and VEH data ${ }^{22}$ also differ by only $0.15 \mathrm{eV}$. Band structure calculations for PTh at LSDA $^{17}$ yield a bandwidth about $1 \mathrm{eV}$ larger than VEH and our method. There is qualitative agreement at all levels of theory that PPy has a larger valence bandwidth than PTh. However, we obtain a smaller difference than solid-state calculations. This is probably caused by different positions of the avoided crossing of the upper two $\pi$-bands. Both PTz and PThTz have smaller valence bandwidths than PPy, but the PTz valence band is still wider than that of PTh.

The conduction bandwidths differ considerably at the various levels of theory. The HF values for PA were obtained with unpolarized basis sets and are very large. With polarization functions HF and MP2 theory yield smaller bandwidths than the DFT/hybrid approach. The LSDA values are in between. Since the numbers are also strongly basis set dependent, one should not overinterpret these results.

Geometries. Table 6 summarizes geometries of the inner rings in sexithiophene and sexipyrrole. Comparison is made with the X-ray structure ${ }^{35}$ of the center rings of sexithiophene in a single crystal and with solid-state LSDA $^{14}$ and HF calculations ${ }^{12,13}$ on PTh and PPy. Horowitz et al. ${ }^{35}$ showed that bond lengths of central rings in terthiophene and sexithiophene differ by no more than $0.01 \AA$, and bond angles by no more than $1^{\circ}$. This indicates that oligomer geometries converge rapidly to polymer structures. It is thus not necessary to perform solid-state calculations to obtain good geometries.

Our optimized structure for sexithiophene differs by less than $0.026 \AA$ and $0.7^{\circ}$ from the sexithiophene crystal structure. The B3P86-30\% oligomer geometry and LSDA solid-state structure of PTh are virtually identical. The $\mathrm{C}-\mathrm{C}$ distance differs by only $0.001 \AA$ in both types of calculations, overestimating the experimental value by 0.026 and $0.025 \AA$ for B3P86-30\% and LSDA, respectively. The only exception is the $\mathrm{C}-\mathrm{S}$ distances, which is $0.023 \AA$ longer at the B3P86-30\% level. The LSDA distance of $1.729 \AA$ is very close to the experimental value. Bond angles differ by up to $0.9^{\circ}$ between B3P86-30\% oligomer and LSDA polymer calculations. Here our data are in closer agreement with experiment than is LSDA.

The agreement between B3P86-30\% oligomer and LSDA solid-state calculations for the polypyrrole is equally good. Again only the $\mathrm{C}$-heteroatom distances differ. For polypyrrole we obtain a $0.026 \AA$ smaller value. The bond angles agree to within $0.9^{\circ}$ in both types of calculations. The agreement between DFT/hybrid and LSDA calculations is very reassuring, since it shows that there is no strong dependence of structural data on the type of functional used.

Compared to $\mathrm{HF}$ results our $\mathrm{C}-\mathrm{X}$ and $\mathrm{C}=\mathrm{C}$ distances are longer. All other parameters are almost identical. The longer $\mathrm{C}=\mathrm{C}$ bonds reflect the increase of conjugation after inclusion of correlation. $\mathrm{C}-\mathrm{X}$ bond lengths are quite sensitive to the level of theory employed. No trend is obvious since HF and DFT/hybrid bond distances are larger for PTh but shorter for PPy than those with LSDA. The $3-2-5^{\prime}$ angle of $113.0^{\circ}$ for PPy in refs 12 and 13 is probably incorrect since it disagrees by $18^{\circ}$ from our and LSDA results and does not fit the series of the data for PTh, polyfuran, and polyselenophene in refs 12 and 13.

\section{Conclusions}

We have shown that a substantial improvement of DFT HOMO-LUMO gaps can be achieved in going beyond LSDA. With DFT/hybrid functionals that include $30 \% \mathrm{HF}$ exchange, vertical excitation energies of medium sized and large oligomers of acetylene, thiophene, pyrrole, and thiazole are reproduced within $\pm 0.25 \mathrm{eV}$ of the corresponding experimental results (solution phase). An exception is terpyrrole, where the difference between theory and experiment amounts to $0.58 \mathrm{eV}$. Extrapolation of oligomer data leads to band gaps within \pm 0.1 $\mathrm{eV}$ of experimental results (solid state).

IPs, EAs, and bandwidths also correlate linearly with inverse chain lengths. We obtained IPs and EAs within $0.8 \mathrm{eV}$ of experimental solution values. The close agreement between our gas-phase IPs and solid-state IPs is, however, due to cancellation between the about $1 \mathrm{eV}$ underestimation with our approach and the solid-state polarization energy. Bandwidths for polyacetylene agree well with VEH results but differ for PTh and PPy.

The B3P86-30\% functional reproduces experimental geometries with excellent accuracy. Moreover, geometries of central rings in sexithiophene and sexipyrrole are in excellent agreement with geometries obtained with solid-state LSDA calculations. This strengthens our conclusion that polymer properties can be obtained from oligomer calculations.

Since hybrid functionals are not available in solid-state codes and may be too time intensive in the near future, a choice has to be made between performing solid-state calculations at lower levels of theory and extrapolating data for molecules obtained at a higher level of theory. The results in this paper show that the latter method leads to better estimates of band gaps. Hybrid functionals were also shown to be superior to local and nonlocal functionals for NMR shift calculations ${ }^{52}$ and for the treatment of charge-transfer complexes. ${ }^{53}$ For both types of problems excitation energies play a crucial role, ${ }^{54}$ and we believe that the improvement is due to the improved description of the energy gap between occupied and virtual orbitals.

Acknowledgment. We would like to thank the Natural Sciences and Engineering Research Council of Canada and Memorial University for financial support and the Computing and Communications Department of Memorial University and Digital Inc. for computing resources.

\section{References and Notes}

(1) André, J.-A.; Delhalle, J.; Brédas, J.-L. Quantum Chemistry Aided Design of Organic Polymers. An Introduction to the Quantum Chemistry of Polymers and its Applications; World Scientific: London, 1991.

(2) Roncali, J. Chem. Rev. 1997, 97, 173 
(3) Dewar, M. J. S.; Zoebisch, E. G.; Healey, E. F.; Stewart, J. J. P. J. Am. Chem. Soc. 1985, 107, 3902.

(4) Hoffmann, R.; Lipscomb, W. N. J. Chem. Phys. 1962, 36, 2179.

(5) Hoffmann, R.; Lipscomb, W. N. J. Chem. Phys. 1962, 37, 2872.

(6) Hoffmann, R. J. Chem. Phys. 1963, 39, 1397.

(7) Su, W. P.; Schrieffer, J. R.; Heeger, A. J. Phys. Rev. Lett. 1979, $42,1698$.

(8) Brédas, J. L.; Chance, R. R.; Silbey, R.; Nicolas, G.; Durand, P. J. Chem. Phys. 1981, 75, 255.

(9) Brédas, J. L.; Chance, R. R.; Silbey, R.; Nicolas, G.; Durand, P. J. Chem. Phys. 1982, 77, 371

(10) Bakhshi, A. K.; Ladik, J.; Seel, M. Phys. Rev. B 1987, 35, 704.

(11) Hunt, W. J.; Goddard, A., III Chem. Phys. Lett. 1969, 3, 414

(12) Otto, P. Int. J. Quantum. Chem. 1994, 52, 353. 97.

(13) Villar, H. O.; Otto, P.; Dupuis, M.; Ladik, J. Synth. Met. 1993, 59

(14) Brocks, G.; Kelly, P. J.; Car, R. Synth. Met. 1993, 55-57, 4243.

(15) Gomes da Costa, P.; Dandrea, R. G.; Conwell, E. M.; Fahlman,

M.; Lögdlund, M.; Stafstrom, S.; Salaneck, W. R.; Graham, S. C.; Friend,

R. H.; Burn, P. L.; Holmes, A. B. Synth. Met. 1993, 55-57, 4320.

(16) Stumpf, R.; Scheffler, M. Comput. Phys. Commun. 1994, 79, 447.

(17) Brocks, G. J. Chem. Phys. 1996, 100, 17327.

(18) Salzner, U.; Lagowski, J. B.; Pickup, P. G.; Poirier, R. A. J. Comput.

Chem. 1997, 18, 1943.

(19) Scheiner, A. C.; Baker, J.; Andzelm, J. W. J. Comput. Chem. 1997, 18,775 .

(20) Lahti, P.; Obrzut, J.; Karasz, F. E. Macromolecules 1987, 20, 2023.

(21) Diaz, A. F.; Crowley, J.; Bargon, J.; Gardini, G. P.; Torrance, J.

B. J. Electroanal. Chem. 1981, 121, 355.

(22) Brédas, J.-L. Electronic Structure of Highly Conducting Polymers. In Handbook of Conducting Polymers; Skotheim, T. A., Ed.; Dekker: New York, 1986; p 859.

(23) Zotti, G.; Martina, S.; Wegner, G.; Schlüter, A.-D. Adv. Mater. 1992, 4, 798.

(24) Parr, P. G.; Yang, W. Density-Functional Theory of Atoms and Molecules; Oxford University Press: New York, 1989.

(25) Dreizler, R. M.; Gross, E. K. U. Density Functional Theory; Springer-Verlag: Berlin, 1990

(26) Fritsche, L. Physica B 1991, 172, 7.

(27) Janak, J. F. Phys. Rev. B 1978, 18, 7165.

(28) Levy, M. Phys. Rev. A 1995, 52, 50.

(29) Nesbet, R. K. J. Phys. Chem. 1996, 100, 6104. 2821.

(31) Godby, R. W.; Schlüter, M.; Sham, L. J. Phys. Rev. B 1988, 37 , 10159.

(32) Williams, A. R.; von Barth, U. Applications of Density Functional Theory to Atoms, Molecules, and Solids. In Theory of the Inhomogeneous Electron Gas; Lundqvist, S., March, N. H., Eds.; Plenum Press: London, 1983.

(33) Baerends, E. J.; Gritsenko, O. V. J. Phys. Chem. A 1997, 101, 5383.

(34) Taliani, C.; Blinov, L. M. Adv. Mater. 1996, 8, 353.

(35) Horowitz, G.; Bachet, B.; Yassar, A.; Lang, P.; Demanze, F.; Fave, J.-L.; Garnier, F. Chem. Mater. 1995, 7, 1337.

(36) Frisch, M. J.; Trucks, G. W.; Schlegel, H. B.; Gill, P. M. W.; Johnson, B. G.; Robb, M. A.; Cheeseman, J. R.; Keith, T.; Petersson, G.
A.; Montgomery, J. A.; Raghavachari, K.; Al-Laham, M. A.; Zakrzewski, V. G.; Ortiz, J. V.; Foresman, J. B.; Peng, C. Y.; Ayala, P. Y.; Chen, W ; Wong, M. W.; Andres, J. L.; Replogle, E. S.; Gomperts, R.; Martin, R. L.; Fox, D. J.; Binkley, J. S.; Defrees, D. J.; Baker, J.; Stewart, J. P.; HeadGordon, M.; Gonzalez, C.; Pople, J. A. Gaussian 94, Revision B.3; Gaussian, Inc.: Pittsburgh, PA, 1995.

(37) Stevens, W.; Basch, H.; Krauss, J. J. Chem. Phys. 1984, 81, 6026.

(38) Frisch, M. J.; Frisch, Æ.; Foresman, J. B. Gaussian 94 User's Reference; Gaussian, Inc.: Pittsburgh, 1994-1995.

(39) Hohenberg, P.; Kohn, W. Phys. Rev. 1964, 136, B864.

(40) Kohn, W.; Sham, L. J. Phys. Rev. 1965, 140, A1133.

(41) Slater, J. C. The Self-Consistent Field for Molecular and Solids. In Quantum Theory of Molecular and Solids; McGraw-Hill: New York, 1974; Vol. 4.

(42) Vosko, S. H.; Wilk, L.; Nusair, M. Can. J. Phys. 1980, 58, 1200

(43) Becke, A. D. J. Chem. Phys. 1993, 98, 5648

(44) Perdew, J. P. Phys. Rev. B 1986, 33, 8822.

(45) Brédas, L.-L.; Silbey, R.; Boudreaux, D. S.; Chance, R. R. J. Am. Chem. Soc. 1983, 105, 6555 .

(46) Colditz, R.; Grebner, D.; Helbig, M.; Rentsch, S. Chem. Phys. 1995, 201, 309.

(47) Martinez, F.; Voelkel, R.; Naegele, D.; Naarmann, H. Mol. Cryst. Liq. Cryst. 1989, 167, 227.

(48) Tani, T.; Grant, P. M.; Gill, W. D.; Street, G. B.; Clarke, T. C. Solid State Commun. 1980, 33, 499.

(49) Kobayashi, M.; Chen, J.; Chung, T.-C.; Moraes, F.; Heeger, A. J.; Wudl, F. Synth. Met. 1984, 9, 77.

(50) Yamamoto, T.; Suganuma, H.; Maruyama, T.; Kubota, K. J. Chem. Soc., Chem. Commun. 1995, 1613.

(51) Sato, N.; Seki, K.; Inokuchi, H. J. Chem. Soc., Faraday Trans. 2 1981, 77, 1621 .

(52) Bühl, M. Chem. Phys. Lett. 1997, 267, 251.

(53) Ruiz, E.; Salahub, D. R.; Vela, A. J. Phys. Chem. 1996, 100, 12265.

(54) Malkin, V. G.; Malkina, O. L.; Casida, M. E.; Salahub, D. R. J. Am. Chem. Soc. 1994, 116, 5898

(55) Hirayama, K. Handbook of Ultrviolett and Visible Absorption Spectra of Organic Compounds; Plenum Press: New York, 1967.

(56) Mosher, O. A.; Flicker, W. M.; Kuppermann, A. J. Chem. Phys. 1973, 59, 6502 .

(57) Flicker, W. M.; Mosher, O. A.; Kuppermann, A. Chem. Phys. Lett. 1977, 45, 492.

(58) Cave, R. J.; Davidson, E. R. J. Phys. Chem. 1988, 92, 2173.

(59) Kutzelnigg, W. Einführung in die Theoretische Chemie, Band 2; Verlag Chemie: Weinheim, 1978

(60) Simmons, W. W. Handbook of Ultraviolet Spectra; Sadler Res. Lab.: Philadelphia, 1979

(61) Keane, M. P.; Naves de Brito, A.; Correira, N.; Svensson, S.; Karlsson, L.; Wannberg, B.; Gelius, U.; Lunell, S.; Salaneck, W. R.; Lögdlund, M.; Swanson, D. B.; MacDiarmid, A. G. Phys. Rev. B 1992, 45, 6390.

(62) Bajic, M.; Humski, K.; Klasinc, L.; Ruscic, B. Z. Naturforsch. B $1985,40,1214$

(63) Willett, G. D.; Baer, T. J. Am. Chem. Soc. 1980, 102, 6774.

(64) Kraak, A.; Wynberg, H. Tetrahedron 1968, 24, 3881

(65) Salaneck, W. R.; Thomas, H. R.; Duke, C. B.; Paton, A.; Plummer, E. W.; Heeger, A. J.; MacDiarmid, A. G. J. Chem. Phys. 1979, 71, 2044. 